\title{
IDOSOS NA UNIVERSIDADE DA MELHOR IDADE: CARACTERISTICAS SOCIAIS, ECONOMICAS, DE SAÚDE E DE UTILIZAÇÃO DE MEDICAMENTOS RELACIONADOS AO SEXO
}

\author{
Roberta Aguiar Brambilla ${ }^{1}$ \\ Elza de Fátima Ribeiro Higa² \\ Carlos Alberto Lazarini ${ }^{3}$
}

resumo

Participantes de Universidades da Terceira Idade possuem perfil de saúde satisfatório, são em sua maioria independentes, com boa cognição e desejo de estabelecer e manter contato social. Porém, prevalece entre eles o uso contínuo de medicamentos. O objetivo deste trabalho foi apresentar o perfil sociodemográfico, socioeconômico, condições de saúde, uso de medicamentos e adesão ao

\footnotetext{
1 Graduada em Enfermagem. Mestre pelo programa "Saúde e Envelhecimento" da Faculdade de Medicina de Marilia - Famema. E-mail: robertaa.brambilla@gmail.com.

2 Graduada em Enfermagem. Doutora em Enfermagem Fundamental (EERP-USP). Docente do Curso de Enfermagem, do Programa de Mestrado Profissional "Ensino em Saúde", do Mestrado Acadêmico "Saúde e Envelhecimento" e do Grupo de Avaliação da Faculdade de Medicina de Marilia - Famema. E-mail: hirifae@@gmail.com.

3 Graduado em Farmácia. Doutor em Farmacologia (USP-SP). Docente do curso de Medicina e do Programa de Mestrado Acadêmico "Saúde e Envelhecimento" da Faculdade de Medicina de Marília - Famema. E-mail: carlos.lazarini@gmail.com.
} 
tratamento de idosos participantes de uma Universidade da Melhor Idade e suas associações com o sexo. Trata-se de uma pesquisa exploratória, analítica, com abordagem quantitativa, em que o sexo foi a variável dependente e os demais dados foram as variáveis independentes. A análise estatística foi a bivariada, com nível de significância onde $p \leq 0,05$. Dos 48 idosos participantes, a maioria era mulher (75\%), vivendo sozinha (66,7\%). Homens apresentaram maior porcentagem de renda de até um salário mínimo (50\%). 0 perfil de uso de medicamentos mostrou: 83,3\% utilizaram algum tipo de medicamento predominando os anti-hipertensivos; $70 \%$ consumiram de um a quatro medicamentos; $80 \%$ dos homens obtêm medicamentos em farmácias e no Sistema Único de Saúde; 100\% dos homens possuem entre média e alta adesão ao tratamento, o que difere estatisticamente das mulheres onde se observou maior porcentagem de baixa adesão (38,9\%). O presente estudo sugere que conhecer o perfil do uso de medicamentos por idosos frequentadores de uma Universidade da Terceira Idade pode subsidiar a inclusão de temas relacionados ao manejo diário de medicamentos, tais como automedicação, adesão terapêutica, entre outros, nas atividades desenvolvidas, visto que a medicação faz parte do dia a dia dessas pessoas.

palavras-chave

Idoso. Medicamento. Universidades.

\section{Introdução}

O avanço cronológico natural leva à utilização de profilaxias e métodos de tratamento, visando à preservação e àmanutenção da saúde. Eventos genéticos, biológicos, psicológicos e sociais contribuem para perdas evolutivas nessa etapa da vida dos indivíduos. Contudo, não impedem que os idosos continuem se desenvolvendo social e intelectualmente (LAURIA; MALTA; DÁTILO, 2015).

Nesse contexto, estudos sobre o envelhecimento e alternativas que proporcionem melhoria da qualidade de vida dos idosos são indispensáveis para um envelhecimento ativo. A longevidade induz mudanças severas no estilo de vida e no perfil da pessoa que envelhece, em nosso país, nas duas últimas décadas. Rejuvenescido, disciplinado, saudável, ativo e jovial são alguns dos adjetivos 
que podem qualificar o cidadão idoso no Brasil contemporâneo (ROZENDO; BORTOLI; DONADONE, 2015).

Nesse processo, nota-se a necessidade de práticas e estratégias adequadas para a continuidade do aprendizado, compartilhando o conhecimento em prol da qualidade de vida do idoso (LIMA-SILVA et al., 2012). Na década de 1990, muitos espaços voltados exclusivamente para a reunião de pessoas idosas foram criados, incluindo grupos de convivência, associações de aposentados, escolas e universidades abertas à terceira idade (LAURIA; MALTA; DÁTILO, 2015).

No que diz respeito às universidades da terceira idade, constituem-se de uma política de atenção ao idoso, que se espalhou globalmente, tornando-se diretamente responsáveis pela ressignificação das experiências do envelhecimento (ROZENDO; BORTOLI; DONADONE, 2015). Assim, as universidades voltadas aos idosos possuem um grande papel na inclusão educacional dessa população, que oferecem atividades que estimulam o autoconhecimento, autorrealização e autoestima (ADAMO et al., 2017).

A primeira Universidade da Terceira Idade (UNATI) surgiu na França, em 1973, seguida pelo Uruguai em 1980. No Brasil, o primeiro programa de atendimento educacional ao idoso numa universidade se inicia em 1982, na Universidade Federal de Santa Catarina (CACHIONI, 2005). Logo após, outras universidades brasileiras criaram UNATIs, dando oportunidades para que as pessoas usufruam da educação na última fase de seu ciclo vital (DÁTILO; BRAGA, 2015).

A Universidade da Melhor Idade (UMI), localizada no Campus de três Lagoas da Universidade Federal do Mato Grosso do Sul, é uma iniciativa do curso de Direito. O projeto possibilita o acesso a cursos, oficinas, atividades curriculares e extracurriculares, no âmbito universitário, com intuito de levar à população idosa as mais diversas áreas do conhecimento de forma diferenciada. Tem como objetivo principal integrar socialmente os idosos, valorizando o conhecimento como meio para que eles possam alcançar a longevidade com qualidade de vida e saúde (BOAVENTURA et al., 2012).

Dados da literatura demonstram a prevalência de mulheres que buscam essas possibilidades de continuidade na construção de conhecimento (ADAMO et al., 2017; ALVES; CEBALLOS, 2018). O predomínio de mulheres em relação aos homens matriculados em UNATIs pode estar relacionado com a predominância feminina e a expectativa de vida da mulher ser mais alta que do homem.

Além disso, outra possível justificativa pelo desinteresse masculino por atividades culturais, educativas e sociais é o preconceito historicamente construído, uma vez que essas atividades sempre foram exercidas por mulheres (ROQUE et al., 2011). Esses autores descrevem que os participantes das UNATIs 
possuem perfil de saúde satisfatório, em sua maioria independentes, com boa cognição e desejo de estabelecer e manter contato social.

Quando se refere à saúde e perfil medicamentoso, tem-se que 78,3\% dos idosos da UNATI de Franca, interior do estado de São Paulo,utilizam medicamentos contínuos (MONTEIRO, B.; MONTEIRO, M., 2013) e a quantidade de medicamentos utilizados por $78,0 \%$ de idosos da UNATI da Universidade Federal de Pernambuco, em Recife,foi de dois ou mais (ALVES; CEBALLOS, 2018).

Possíveis causas são estudadas com relação ao sexo, sendo que mulheres idosas têm maior tendência (85,9\%) à se automedicarem, segundo dados obtidos com idosos frequentadores de um centro de referência na Atenção à Saúde do Idoso de um hospital público de ensino de Belo Horizonte (OLIVEIRA, S. et al., 2018), pois a figura da mulher está relacionada com o papel social, preocupação com a saúde da família, tendo acesso mais a farmácias e, consequentemente, aos medicamentos de balcão (NASCIMENTO; VALDÃO, 2012).

De acordo com Lobo (2015), uma maior utilização de medicamentos pelas mulheres idosas pode estar relacionada com aspectos de ordem biológica (mulheres são mais afetadas por problemas de saúde não fatais), sociocultural (mulheres utilizam mais os serviços de saúde e estão mais familiarizadas com os medicamentos) e psicológica (consciência quanto aos seus sintomas físicos e mais atenção sobre os seus problemas de saúde).

Considerando o crescente envelhecimento da população brasileira, o fato da sociedade se organizar e propiciar diversosespaços para esses idosos continuarem seu processo de formação - dentre eles, a Universidade Aberta da Terceira Idade - e a crescente utilização de medicamentos nessa faixa etária, este trabalho foi motivado pelo interesse em obter o perfil medicamentoso dos participantes de uma Universidade Aberta à Terceira Idade a partir de seu contexto social.

Frente ao exposto, o objetivo do presente estudo foi apresentar o perfil sociodemográfico, socioeconômico, condições de saúde, uso de medicamentos e adesão ao tratamento de idosos participantes de uma Universidade Aberta da Terceira Idade em Três Lagoas, Mato Grosso do Sul, denominada Universidade da Melhor Idade (UMI) e suas associações com o sexo.

\section{Método}

Trata-se de uma pesquisa exploratória e analítica, com abordagem quantitativa. O estudo foi realizado com 48 idosos, com idade igual ou superior a 60 anos, participantes de uma UMI, desenvolvida por uma universidade federal, 
localizada no interior do Estado de Mato Grosso do Sul. As atividades da UMI são semanais e enfocam as áreas de humanas, exatas e da saúde e os idosos podem optar por uma ou mais delas.

Para a coleta de dados, foi utilizado um formulário autoaplicado. Todos os idosos regularmente matriculados na UMI, no ano de 2016, foram convidados a participarem da pesquisa. A variável dependente foi o sexo. As variáveis independentes foram as demográficas (idade, estado conjugal, religião e etnia), socioeconômicas (classe social e renda familiar), condições de saúde (número de morbidades, de consultas, de internações e autopercepção de saúde. A autopercepção de saúde foi avaliada de acordo com Confortin et al. (2015). Esses autores agruparam, para análise estatística, como autoavaliação positiva as respostas "regular", "boa" e "ótima" e como autoavaliação negativa as respostas "ruim" e "péssima". Além disso, também foi avaliado operfil medicamentoso (uso de medicamentos, quantidade utilizada, local de obtenção, gastos, prática de automedicação e adesão ao tratamento). Para a classificação econômica, utilizou-se o Critério de Classificação Econômica Brasil (ABEP, 2014). Os medicamentos utilizados pelos idosos foram classificados de acordo com a Anatomical Therapeutical Chemical Classification System (ATC) (WHO, 2013). Para a adesão ao uso de medicamentos, foi utilizada a versão em português do Teste de Morisky-Green (MORISKY; GREEN; LEVINE, 1986).

Os dados foram processados e analisados com a utilização do software SPSS, versão 17.0. Foram obtidas as frequências absolutas e relativas, e comparadas às variáveis selecionadas no estudo para verificação da existência de associação, utilizando-se o Teste do Qui-quadrado ou o Teste Exato de Fisher. Foram consideradas estatisticamente significativas as relações entre variáveis, em que $\mathrm{p} \leq 0,05$.

Em cumprimento da Resolução 466/12 do Conselho Nacional de Saúde, a pesquisa foi aprovada pelo Comitê de Ética em Pesquisa (CEP) envolvendo seres humanos da Faculdade de Medicina de Marília, sob parecer n.ํㅜ․088.542. Os participantes assinaram o Termo de Consentimento Livre e Esclarecido antes do preenchimento do questionário.

\section{Resultados}

Dos 71 idosos regularmente matriculados na UMI, em 2016, 61 estavam presentes no dia da apresentação do presente trabalho. Desses, quatro relataram não saber escrever, negando-se a responderem as questões, e sete também recusaram a participação por terem chegado atrasados e demonstrarem 
desinteresse. Totalizaram-se 50 idosos ativos e alfabetizados no preenchimento dos questionários. O número total de respondentes foi de 48 idosos, pois dois entregaram o questionário respondido de forma incompleta, o que inviabilizou a inclusão dos mesmos no trabalho.

Dos 48 entrevistados, 36 (75\%) eram mulheres. O grupo etário prevalente foi de 60-69 anos em ambos os sexos, sendo cinco homens (41,7\%) e 19 mulheres (52,8\%). A análise estatística mostrou associação significativa entre estado conjugal e sexo $(\mathrm{p}=0,05)$, em que "viver sozinho" é predominante entre as mulheres $(n=24 ; 66,7 \%)$. Mais da metade dos entrevistados são católicos ( $n=$ $37 ; 77,1 \%)$ e de raça branca $(n=29 ; 60,4 \%$ ) (Tabela 1$)$.

Tabela 1 - Variáveis sociodemográficas dos idosos matriculados na Universidade da Melhor Idade, Três Lagoas, 2016

\begin{tabular}{|c|c|c|c|c|c|}
\hline \multirow{2}{*}{ Variáveis } & \multicolumn{2}{|c|}{ Masculino - 12} & \multicolumn{2}{|c|}{ Feminino - 36} & \multirow[t]{2}{*}{$p$} \\
\hline & $\mathrm{N}$ & $\%$ & $\mathrm{~N}$ & $\%$ & \\
\hline Idade (anos) & & & & & 0,24 \\
\hline $60-69$ & 5 & 41,7 & 19 & 52,8 & \\
\hline $70-79$ & 3 & 25,0 & 13 & 36,1 & \\
\hline 80 ou mais & 4 & 33,3 & 4 & 11,1 & \\
\hline Estado conjugal & & & & & $0,05^{\star}$ \\
\hline Vive só & 4 & 33,3 & 24 & 66,7 & \\
\hline Vive acompanhado & 8 & 66,7 & 12 & 33,3 & \\
\hline Religião & & & & & 0,12 \\
\hline Católica & 8 & 66,7 & 29 & 80,6 & \\
\hline Evangélica & 4 & 33,3 & 3 & 8,3 & \\
\hline Espírita & 0 & 0 & 2 & 5,6 & \\
\hline Outras & 0 & 0 & 2 & 5,6 & \\
\hline Etnia & & & & & 0,11 \\
\hline Branco & 8 & 66,7 & 21 & 58,3 & \\
\hline Preto & 3 & 25,0 & 2 & 5,6 & \\
\hline Amarelo & 0 & 0 & 1 & 2,8 & \\
\hline Pardo & 1 & 8,3 & 12 & 33,3 & \\
\hline
\end{tabular}

Fonte: Elaborada pelos autores.

Nota: ${ }^{\star}$ Teste Exato de Fisher $-p \leq 0,05$. 
No que se refere às variáveis socioeconômicas, há um predomínio de homens na classe " $B$ " ( $n=5 ; 41,7 \%)$ e mulheres na " $C$ " $(n=19 ; 52,8 \%)$. Mais da metade dos participantes são aposentados/pensionistas ( $\mathrm{n}=33 ; 68,7 \%)$, com renda familiar predominante de até um salário mínimo no sexo masculino $(\mathrm{n}=6 ; 50 \%)$ e 13 mulheres declaradas sem renda (36,1\%). A análise estatística mostrou associação positiva entre esse parâmetro e sexo $(p=0,03)$ (Tabela 2).

Tabela 2 - Dados socioeconômicos dos idosos matriculados na Universidade da Melhor Idade, Três Lagoas, 2016

\begin{tabular}{|c|c|c|c|c|c|}
\hline \multirow{2}{*}{ Variáveis } & \multicolumn{2}{|c|}{ Masculino-12 } & \multicolumn{2}{|c|}{ Feminino - 36} & \multirow[t]{2}{*}{$p$} \\
\hline & $\mathrm{N}$ & $\%$ & $\mathrm{~N}$ & $\%$ & \\
\hline Classe social & & & & & 0,60 \\
\hline A & 0 & 0 & 1 & 2,8 & \\
\hline $\mathrm{B}$ & 5 & 41,7 & 12 & 46,2 & \\
\hline C & 4 & 33,3 & 19 & 52,8 & \\
\hline $\mathrm{D}$ & 1 & 8,3 & 2 & 5,6 & \\
\hline Não respondeu & 2 & 16,7 & 2 & 5,6 & \\
\hline Situação trabalhista & & & & & 0,17 \\
\hline Aposentado/pensionista & 11 & 91,7 & 21 & 58,3 & \\
\hline Desempregado & 1 & 8,3 & 4 & 11,1 & \\
\hline Dona de casa & 0 & 0 & 5 & 13,9 & \\
\hline Aposentado/pensionista e Desempregado & 0 & 0 & 1 & 2,8 & \\
\hline Aposentado/pensionista e Dona de casa & 0 & 0 & 3 & 8,3 & \\
\hline Desempregado e Dona de casa & 0 & 0 & 2 & 5,6 & \\
\hline Renda familiar (salário mínimo - SM) & & & & & $0,03^{*}$ \\
\hline Sem renda & 3 & 25,0 & 13 & 36,1 & \\
\hline Até $1 \mathrm{SM}$ & 6 & 50,0 & 9 & 25,0 & \\
\hline De 1 até 2 SM & 0 & 0 & 8 & 22,2 & \\
\hline De 2 até 3 SM & 1 & 8,3 & 3 & 8,3 & \\
\hline De 3 até 5 SM & 2 & 16,7 & 0 & 0 & \\
\hline De 5 até $10 \mathrm{SM}$ & 0 & 0 & 2 & 5,6 & \\
\hline De 10 até 20 SM & 0 & 0 & 1 & 2,8 & \\
\hline
\end{tabular}

Fonte: Elaborada pelos autores.

Nota: *Teste do Qui-quadrado - p $\leq 0,05$. 
Os homens apresentam predomínio de uma morbidade ( $\mathrm{n}=6 ; 50 \%)$. A maior parte das mulheres $(n=10 ; 27,8 \%$ ) passou por pelo menos três consultas médicas nos últimos seis meses antecedentes à entrevista, enquanto a maioria dos homens ( $\mathrm{n}=5 ; 41,7 \%$ ) passou por uma consulta neste período, com ausência de internações hospitalares na maioria, em ambos os sexos ( $n=45$; 93,7\%). A autoavaliação de saúde foi considerada positiva na maior parte dos entrevistados ( $\mathrm{n}=46 ; 95,8 \%$ ) (Tabela 3$)$.

Tabela 3 - Condições de saúde dos idosos matriculados na Universidade da Melhor ldade, Três Lagoas, 2016

\begin{tabular}{|c|c|c|c|c|c|}
\hline \multirow{2}{*}{ Variáveis } & \multicolumn{2}{|c|}{ Masculino - 12} & \multicolumn{2}{|c|}{ Feminino - 36} & \multirow[t]{2}{*}{$p$} \\
\hline & $\mathrm{N}$ & $\%$ & $\mathrm{~N}$ & $\%$ & \\
\hline Quantidade de morbidades & & & & & 0,08 \\
\hline Nenhuma & 0 & 0 & 4 & 11,1 & \\
\hline 1 & 6 & 50,0 & 7 & 19,4 & \\
\hline 2 & 1 & 8,3 & 6 & 16,7 & \\
\hline 3 & 4 & 33,3 & 7 & 19,4 & \\
\hline 4 & 1 & 8,3 & 7 & 19,4 & \\
\hline $5 \mathrm{ou}+$ & 0 & 0 & 5 & 13,9 & \\
\hline Número de consultas & & & & & 0,38 \\
\hline Nenhuma & 0 & 0 & 5 & 13,9 & \\
\hline 1 & 5 & 41,7 & 8 & 22,2 & \\
\hline 2 & 2 & 16,7 & 5 & 13,9 & \\
\hline 3 & 2 & 16,7 & 10 & 27,8 & \\
\hline 4 & 0 & 0 & 1 & 2,8 & \\
\hline $5 \mathrm{ou}+$ & 3 & 25,0 & 7 & 19,4 & \\
\hline Internações & & & & & 0,57 \\
\hline Sim & 1 & 8,3 & 2 & 5,6 & \\
\hline Não & 11 & 91,7 & 34 & 94,4 & \\
\hline Como considera a saúde & & & & & 0,44 \\
\hline Positiva & 11 & 91,7 & 35 & 97,2 & \\
\hline Negativa & 1 & 8,3 & 1 & 2,8 & \\
\hline
\end{tabular}

Fonte: Elaborada pelos autores. 
De todos os entrevistados, 40 (83,3\%) consumiram algum tipo de medicamento nos últimos quinze dias que antecederam à entrevista. Desses, 28 $(58,3 \%)$ consumiram de um a quatro medicamentos. Quanto à forma de acesso aos medicamentos, oito homens ( $80 \%$ ) obtêm em farmácias ou na associação entre a rede do Sistema Único de Saúde e farmácias, enquanto entre as mulheres há uma prevalência de obtenção apenas nas farmácias ( $n=19 ; 63,3 \%)$. Os gastos referentes aos medicamentos mostram que 11 mulheres $(30,6 \%)$ despendem de $\mathrm{R} \$ 50,00$ a $\mathrm{R} \$ 100,00$, enquanto que quatro homens $(33,3 \%)$ têm um gasto médio de $\mathrm{R} \$ 100,00$ a $\mathrm{R} \$ 200,00$. Os entrevistados, em sua maioria $(\mathrm{n}=27$; $56,2 \%$ ), afirmam não utilizar receitas caseiras, e quanto à automedicação, metade dos homens $(n=6,50 \%)$ confirma essa prática. No que diz respeito à adesão ao tratamento medicamentoso, seis homens (50\%) possuem entre média e alta adesão, enquanto 14 mulheres (38,9\%), baixa adesão. A análise estatística mostrou associação positiva entre esse parâmetro e sexo $(p=0,01)$ (Tabela 4).

Tabela 4 - Uso de medicamentos pelos idosos matriculados na Universidade da Melhor Idade, Três Lagoas, 2016

\begin{tabular}{|c|c|c|c|c|c|}
\hline \multirow{2}{*}{ Variáveis } & \multicolumn{2}{|c|}{ Masculino - 12} & \multicolumn{2}{|c|}{ Feminino - 36} & \multirow[t]{2}{*}{$p$} \\
\hline & $\mathrm{N}$ & $\%$ & $\mathrm{~N}$ & $\%$ & \\
\hline Uso de medicamentos & & & & & 0,64 \\
\hline Sim & 10 & 83,3 & 30 & 83,3 & \\
\hline Não & 2 & 16,7 & 6 & 16,7 & \\
\hline Quantos medicamentos & & & & & 0,47 \\
\hline $1-4$ & 7 & 70,0 & 21 & 70,0 & \\
\hline $5 \mathrm{ou}+$ & 3 & 30,0 & 9 & 30,0 & \\
\hline Onde obtém os medicamentos & & & & & 0,22 \\
\hline SUS & 2 & 20,0 & 7 & 23,3 & \\
\hline Farmácia & 4 & 40,0 & 19 & 63,3 & \\
\hline SUS + Farmácia & 4 & 40,0 & 3 & 10,0 & \\
\hline Farmácia + Terceiros & 0 & 0 & 1 & 3,3 & \\
\hline
\end{tabular}




\begin{tabular}{|c|c|c|c|c|c|}
\hline \multirow{2}{*}{ Variáveis } & \multicolumn{2}{|c|}{ Masculino - 12} & \multicolumn{2}{|c|}{ Feminino - 36} & \multirow[t]{2}{*}{$p$} \\
\hline & $\mathrm{N}$ & $\%$ & $\mathrm{~N}$ & $\%$ & \\
\hline Gasto com medicamentos (Reais) & & & & & 0,78 \\
\hline$<50$ & 1 & 8,3 & 6 & 16,7 & \\
\hline $50-100$ & 3 & 25,0 & 11 & 30,6 & \\
\hline $100-200$ & 4 & 33,3 & 9 & 25,0 & \\
\hline $200-300$ & 0 & 0 & 2 & 5,6 & \\
\hline $300-400$ & 1 & 8,3 & 3 & 8,3 & \\
\hline $400-500$ & 3 & 25,0 & 3 & 8,3 & \\
\hline Mais de 500 & 0 & 0 & 2 & 5,6 & \\
\hline Automedicação & & & & & 0,36 \\
\hline $\operatorname{Sim}$ & 6 & 50,0 & 14 & 38,9 & \\
\hline Não & 6 & 50,0 & 22 & 61,1 & \\
\hline Grau de adesão ao tratamento & & & & & $0,01^{*}$ \\
\hline Alta adesão & 6 & 50,0 & 10 & 27,8 & \\
\hline Média adesão & 6 & 50,0 & 12 & 33,3 & \\
\hline Baixa adesão & 0 & 0 & 14 & 38,9 & \\
\hline
\end{tabular}

Fonte: Elaborada pelos autores.

Nota: *Teste do Qui-quadrado - p $\leq 0,05$.

Em relação à quantidade total de medicamentos utilizados pelos idosos, independente do sexo e, de acordo com a classificação por sistemas anatômicos, é possível observar predomínio de medicamentos utilizados para tratamento de doenças do aparelho cardiovascular $(n=33 ; 50,8 \%)$, sendo os anti-hipertensivos os mais utilizados $(n=13 ; 20 \%)$ (Tabela 5$)$. 
Tabela 5 - Classe de medicamentos segundo a Anatomical Therapeutical Chemical Classification System (ATC) utilizados pelos idosos matriculados na Universidade da Melhor Idade, Três Lagoas, 2016

\begin{tabular}{|c|c|c|}
\hline Classificação Anatômica/Grupo farmacológico & $\mathrm{n}$ & $\%$ \\
\hline \multicolumn{3}{|l|}{ Aparelho cardiovascular } \\
\hline Anti-hipertensivo & 13 & 20,0 \\
\hline Hipolipemiantes & 6 & 9,2 \\
\hline Antiarrítmicos & 1 & 1,5 \\
\hline Betabloqueadores & 7 & 10,8 \\
\hline Diuréticos & 5 & 7,7 \\
\hline Outros & 1 & 1,5 \\
\hline \multicolumn{3}{|l|}{ Aparelho digestório e metabolismo } \\
\hline Vitaminas & 2 & 3,1 \\
\hline Hipoglicemiantes orais & 6 & 9,2 \\
\hline Medicamentos relacionados à acidez & 2 & 3,1 \\
\hline Outros & 3 & 4,6 \\
\hline \multicolumn{3}{|l|}{ Sistema nervoso } \\
\hline Antiepiléticos & 2 & 3,1 \\
\hline Vasodilatadores & 3 & 4,6 \\
\hline \multicolumn{3}{|l|}{ Sistema musculoesquelético } \\
\hline Anti-inflamatórios e antirreumáticos & 7 & 10,8 \\
\hline \multicolumn{3}{|l|}{ Sangue e órgãos hematopoiéticos } \\
\hline Medicamentos antitrombóticos & 1 & 1,5 \\
\hline \multicolumn{3}{|l|}{ Preparações hormonais sistêmicas } \\
\hline Preparações da tireoide & 3 & 4,6 \\
\hline \multicolumn{3}{|l|}{ Órgãos sensoriais } \\
\hline Oftalmológicos (lubrificantes e hipotensores oculares) & 1 & 1,5 \\
\hline \multicolumn{3}{|l|}{ Aparelho geniturinário e hormônios sexuais } \\
\hline Estrogênios & 2 & 3,1 \\
\hline Total & 65 & 100 \\
\hline
\end{tabular}


Houve predominância de participação das mulheres na pesquisa. Ao avalia participantes de uma UNATI em Goiânia, no estado de Goiás, 90,0\% eram mulheres (ADAMO et al., 2017). A maior participação de mulheres em atividades de Universidade da Terceira Idade também esteve presente em duas cidades do interior do estado de São Paulo, São Carlos e Marília, com 89\% (INOUYE et al., 2018) e 72\% (DÁTILO; MARIN, 2015), respectivamente. Os dados do presente estudo mostram menor participação masculina na UMI, possivelmente justificada pelo fato de que os homens procuram menos esse tipo de programa (ORDONEZ; CACHIONI, 2011).

No que tange à idade, a maior porcentagem tanto de homens quanto de mulheres se encontra no intervalo entre 60 e 69 anos, faixa etária essa também prevalente em outros estudos, envolvendo idosos frequentadores de Universidades da Terceira Idade (ADAMO et al., 2017; ALVES; CEBALLOS, 2018; SANTOS, A.; NOGUEIRA; BORJA-OLIVEIRA, 2018).

Neste estudo, os dados demográficos dos entrevistados demonstram diferença significativa quanto ao estado conjugal, em que a maioria das mulheres vive sozinha, dados esses semelhantes a outros estudos (ALVES; CEBALLOS, 2018; PAULA JÚNIOR et al., 2013). A expectativa de vida pode justificar esses dados. Em 2012, a mesma era de 71 anos para o sexo masculino e 74,6 para o sexo feminino, já que homens morrem mais cedo e existe um número considerável de mulheres viúvas, idosas que frequentemente optam pela vida sem um novo companheiro, por ainda se submeterem às normas e regras sociais ou pela família não declarar apoio para novos relacionamentos amorosos (SOUZA et al., 2015).

A religião à qual o entrevistado pertence, não mostrou diferenças significativas entre os sexos. $\mathrm{O}$ ato de buscar uma religião traz resultados positivos tanto para a saúde física quanto à mental em idosos que residem sozinhos (COSTA, F.; NAKATA; MORAIS, 2015) ou em comunidade (ABDALA et al., 2015), além disso, a prática religiosa pode se configurar como possibilidade para o enfrentamento de mulheres diante de sua condição histórica de dominação, fortalecendo-a dentro de sua família ou em outras relações sociais (FARIA; DAVID; ROCHA, 2011).

Ao referir-se a raça/cor dos entrevistados, mais da metade dos participantes informaram ser de cor branca, semelhante aos estudos de Haymussi (2015) e Borim, Barros e Neri (2012). Já para Alves (2014), a raça parda foi predominante com a população participante da UNATI de Pernambuco. Estes dados justificam-se pela mistura de raças/cor ser característica do nosso país. 
Mais da metade dos participantes são aposentados/pensionistas, dados relativamente semelhantes a um estudo realizado em uma UNATI de Maringá - PR (LOLLI; MAIO, 2015). De acordo com M. Rodrigues (2015, p. 776), “o aposentado vive uma angústia com essa apropriação do seu tempo, pois tem uma sensação de liberdade por não ter compromissos com horário, mas também vem o medo de sentir-se inútil para a sociedade". Essa autora traz, ainda, que o tempo livre conquistado a partir da aposentadoria, permitiu a inserção em outras atividades prazerosas, tais como, iniciar atividades junto a UNATI. Essa procura por ocupação do tempo livre se dá principalmente pelas mulheres (LOLLI; MAIO, 2015).

Vale ressaltar que, dos participantes entrevistados no presente estudo, a maioria dos homens pertencia à classe social "B", enquanto a maioria das mulheres era da classe " $C$ ". A renda familiar predominante foi de até um salário mínimo para sexo masculino e mulheres declaradas sem renda no total de 36,1\%. Haymussi (2015) relata renda mensal preponderante de dois a três salários mínimos em seu estudo na UNATI de Caçador, interior de Santa Catarina. Porém, o mesmo autor relata que houve um número expressivo de idosos que recebe um salário mínimo. A renda de até um salário mínimo é encontrada em demais estudos (KINOSHITA et al., 2012; ALVES, 2014). Almeida et al. (2015), em estudo que analisou a contribuição da renda de idosas no orçamento familiar, mostrou que $40 \%$ das mesmas moram com familiares que dependiam de seus rendimentos, tais como filhos, netos ou o cônjuge. Esses mesmos autores trazem que a renda do idoso faz parte do orçamento doméstico, principalmente em situações de desemprego dos filhos.

Ao se tratar de morbidades, Tavares et al. (2013), em trabalho realizado com idosos, independentemente do sexo, apontam que metade dos entrevistados relatou ter duas ou mais morbidades. As mais frequentes foram: hipertensão arterial (55,3\%), problemas na coluna $(37,4 \%)$, problemas no coração $(29,6 \%)$ e diabetes $(15,1 \%)$. Cerca de $1 / 3$ apresentava incapacidades instrumentais para a vida diária, 14,9\% apresentaram sinais de depressão e 13,4\% de déficit cognitivo.

Nossos resultados apontam que a maioria das mulheres $(63,9 \%)$ passaram por pelo menos de uma atrês consultas nos últimos seis meses antecedentes à entrevista, enquanto a maior parte dos homens (41,7\%) possuem em torno de uma consulta neste período, com ausência de internações hospitalares na maioria ( $n=45,93,7 \%)$, em ambos os sexos, os quais caracterizam como positiva a sua saúde $(\mathrm{n}=46,95,8 \%)$.

Em relação à percepção de saúde quanto a morbidades relatadas pelos entrevistados, mulheres apresentam em média de uma a cinco morbidades. Kinoshita et al. (2012), ao avaliarem a autopercepção de saúde em idosos que 
frequentam uma UNATI, observaram que a presença de doenças crônicas exerceu influência negativa na percepção de saúde de indivíduos idosos, uma vez que os participantes, portadores de três ou mais doenças crônicas, apresentaram pior satisfação com a vida, principalmente, nos domínios da saúde e da capacidade física.

No presente estudo, mesmo as mulheres relatando ter mais de três morbidades, consideram sua saúde como positiva, contrapondo o apresentado anteriormente. Já a maioria dos homens apresentou coerência entre considerar sua saúde como positiva e ter apenas uma morbidade. Esses dados se assemelham aos observados com idosos longevos (80 anos ou mais), residentes na comunidade (ANDRADE, L. et al., 2018).

De todos os entrevistados, 83,3\% consumiram algum tipo de medicamento nos últimos quinze dias que antecederam à entrevista, com predomínio dos anti-hipertensivos. O consumo total de medicamentos se assemelha ao observado com idosos matriculados na Universidade da Terceira Idade, da Universidade de Franca, SP, onde 78,3\% dos entrevistados fizeram uso de algum medicamento (MONTEIRO, B.; MONTEIRO, M., 2013), bem como em estudos realizados em diferentes regiões do país (MUNIZ et al., 2017; COSTA, C. et al., 2017; SMANIOTO; HADDAD, 2013). Essas altas porcentagens de utilização de medicamentos estão normalmente associadas ao envelhecimento acompanhado do aumento da presença de morbidades nessa faixa etária.

Além da terapia medicamentosa contínua, tem-se a preocupação quanto à classe destes medicamentos a serem utilizados. A Sociedade Americana de Geriatria (AGS), por meio do Critério de Beers, analisa as medicações potencialmente inapropriadas (MPIs) para o uso pelos idosos. Trata-se de uma lista explícita de MPIs a serem evitados por essa faixa etária, e em indivíduos com certas doenças ou síndromes, são prescritos em doses reduzidas, com precaução e cuidadosamente monitorados (AGS, 2015).

Trabalho realizado em um centro de convivênciapara idosos em Campo Grande, MS, mostrou que 39,6\% dos participantesutilizaram pelo menos um MPI (RIGHES, 2015). T. Santos et al. (2013), em estudo com 934 idosos, descrevem que $24,6 \%$ dos mesmos consomem pelo menos um medicamento impróprio, $90,2 \%$ provenientes de receita médica atual, sendo benzodiazepínicos de meia vida longa e os antidepressivos, os mais consumidos pelos entrevistados. Em nosso estudo, não houve apresentação de consumo de MPIs pelos entrevistados. Supõe-se que os profissionais de saúde prescritores podem estar levando em consideração o critério de Beers para receitarem os medicamentos para esse grupo específico de idosos. 
A prevalência de compra de medicamentos em farmácias convencionais é observada, com pouco acesso gratuito (VIANA et al., 2015). Esses dados divergem dos apresentados por Matta et al. (2018) em estudo que avaliou o local de obtenção de medicamentos por usuários do SUS. Esses autores mostram que essa população tem como principal fonte de medicamentos o próprio SUS, todavia, há também a presença de outros locais de obtenção de medicamentos, sugerindo dificuldades ao acesso dentro do SUS.

Dados da literatura confirmam a prática de automedicação pelos idosos (OLIVEIRA, M. et al., 2012; SANTOS, T. et al., 2013). De 355 idosos estudados por B. Rodrigues (2013), a prevalência do uso de medicamentos na última semana antecedente à entrevista foi igual a 80,3\%, sendo a polimedicação verificada em $19,3 \%$ e a automedicação em $13 \%$.

A prevalência do sexo feminino na automedicação também é afirmada nos estudos (MONTEIRO, S.; AZEVEDO; BELFORT, 2014; RODRIGUES, B., 2013; SANTOS, T. et al., 2013). A. Santos, Nogueira, Borja-Oliveira (2018), em estudo realizado com idosos que frequentam uma Universidade Aberta da Terceira Idade, na capital paulista, mostraram que dos 138 frequentadores, 82 (59,4\%) declararam a prática de automedicação, justificando a mesma por experiência anterior com o uso do medicamento e a certeza de que o mesmo é seguro. Vale ressaltar que a maioria $(75,4 \%)$ dos entrevistados era do sexo feminino.

Em relação à adesão ao tratamento medicamentoso, o predomínio da baixa adesão observado em mulheres diverge do encontrado na literatura, em que demonstra que homens apresentam maior dificuldade de aderir ao tratamento medicamentoso (ANDRADE, D. et al., 2011), ou que não há diferenças na adesão em relação ao sexo (GIROTTO et al., 2013). Existem diversos fatores que influenciam a não adesão ao tratamento medicamentoso na população idosa. Dentre eles, podemos destacar: o esquecimento e o atraso no uso (STEFANO et al., 2017), presença simultânea de várias morbidades associadas à polimedicação, ausência de plano de saúde, baixa escolaridade e perda de independência e da capacidade cognitiva (MUNIZ et al., 2017). Sardinha et al. (2015) mostram dois fatores como principais responsáveis pela não adesão medicamentosa: a falta de medicamentos nas Unidades Básicas de Saúde; e a baixa renda familiar mensal informada. Além desses fatores, também é possível observar que a idade, ter que comprar (totalmente ou em parte) os seus medicamentos, possuir incapacidade instrumental para a vida diária e usar três ou mais medicamentos podem estar associados significativamente à baixa adesão (TAVARES et al., 2013).

A compreensão da falta de adesão ao tratamento medicamentoso requer esforços dos profissionais de saúde, uma vez que envolvem fatores sociais, 
econômicos e culturais e aqueles relacionados ao funcionamento dos serviços, às doenças de base, às comorbidades, ao tratamento e, finalmente, ao comportamento dos pacientes (STEFANO et al., 2017) sendo um desafio a ser alcançado, pois os mecanismos envolvidos no comportamento dos indivíduos são complexos (SARDINHA et al., 2015).

Por fim, o fato do presente trabalho ter sido realizado em um único local é reconhecido como limitação, comprometendo a generalização dos achados. Nesse sentido, sugere-se que haja mais estudos sobre o uso de medicamentos em idosos que frequentam Universidades Abertas da Terceira Idade.

\section{Conclusão}

No presente estudo foi possível evidenciar diferenças significativas entre diversas variáveis e sexo de idosos que frequentam a Universidade da Melhor Idade.

O predomínio de mulheres que vivem sós e homens que vivem acompanhados no presente estudo pode ser justificado pela maior longevidade da mulher, sua opção por manter-se sozinha após a viuvez e a adoção de práticas cada vez mais independentes.

A renda familiar com variação de um a dois salários mínimos é uma realidade brasileira, inclusive com a participação do idoso nesta renda. Observa-se, também, no presente estudo, que a maioria dos entrevistados são aposentados e contribuem com sua aposentadoria no orçamento familiar.

Os alunos da Universidade da Melhor Idade da Universidade Federal do Mato Grosso do Sul apresentam perfil semelhante ao uso de medicamentos quando comparados aos de outras universidades da terceira idade, inclusive com o predomínio do uso de anti-hipertensivos. Ao se tratar do grau de adesão dos medicamentos e suas características, o uso contínuo de medicamentos é predominante também em demais estudos. Pode-se considerar que a baixa adesão intencional encontrada principalmente pelas mulheres deve-se ao fato de como as mesmas consideram sua saúde, seus efeitos colaterais, aumento de morbidades e a apropriação de informações quanto à sua doença.

O conhecimento do perfil do uso de medicamentos por idosos frequentadores de uma universidade aberta da terceira idade pode subsidiar propostas de inclusão de temas como automedicação, adesão terapêutica, entre outros, tais como armazenamento adequado de medicamentos, nas atividades desenvolvidas por estas universidades, objetivando melhorar os conhecimentos dos idosos sobre a utilização de medicamentos, prática essa presente no dia a dia dessas pessoas. 
OLDER ADULTS AT THE UNIVERSITY:

GENDER DIFFERENCES IN SOCIAL,

ECONOMIC, HEALTH AND USE OF MEDICATION

abstract

Older adults enrolled in universities have a satisfactory health profile, mostly independent, with good cognition and desire to establish and maintain social contact. However, the use of continuous medications prevails. The objective of this work was to present the socio-demographic, socioeconomic, health conditions, use of medications and adherence to the treatment profile of older participants of a university of best age and association with gender. This is an exploratory, analytical research with a quantitative approach, where gender was the dependent variable and the other data were the independent variables. The statistical analysis was bivariate, with significance level where $\mathrm{p} \leq 0.05$. Of the 48 older participants, most were women (75\%), living alone (66.7\%). Men had a higher income percentage of up to a minimum wage (50\%). The drug use profile showed: 83.3\% used some type of medication predominating antihypertensive drugs; $70 \%$ consumed from one to four drugs; $80 \%$ of the men obtain medications in pharmacies and SUS; and 100\% of the men have between mean and high adherence to treatment, which differs statistically from women, where there was a higher percentage of low adherence (38.9\%). The present study suggests that knowing the profile of the use of medications by older people attending at third age university can subsidize the inclusion of topics related to daily management of medications such as self-medication, therapeutic adherence, among others, in the activities developed, since the medication is part of the day to day of these people.

keywords

Older Adults. Medicine. Universities.

referências

ABDALA, Gina et al. Religiosidade e qualidade de vida relacionada à saúde do idoso. Revista de Saúde Pública, São Paulo, v. 49, n. 55, p. 1-9, 2015.

ADAMO, Chadi et al. Universidade aberta para a terceira idade: o impacto da educação continuada na qualidade de vida dos idosos. Revista Brasileira de Geriatria e Gerontologia, Rio de Janeiro, v. 20, n. 4, p. 550-560, 2017. 
ALMEIDA, Alessandra et al. A feminização da velhice: em foco as características socioeconômicas, pessoais e familiares das idosas e o risco social. Textos \& Contextos, Porto Alegre, v. 14, n. 1, p. 115-131, 2015.

ALVES, Niedja. Polifarmácia em idosos do programa Universidade Aberta à Terceira Idade da Universidade Federal de Pernambuco. 2014. Dissertação (Mestrado em Saúde Coletiva) - Universidade Federal de Pernambuco, Recife, 2014

ALVES, Niedja; CEBALLOS, Albanita. Polifarmácia em idosos do programa universidade aberta à terceira idade. Journal of Health \& Biological Sciences, Fortaleza, v. 6, n. 4, p. 412-418, 2018.

AMERICAN GERIATRICS SOCIETY (AGS). Updated Beers Criteria for Potentially Inappropriate Medication Use in Older Adults. Journal of the American Geriatrics Society, New York, v. 63, n. 11, p. 2227-2246, 2015.

ANDRADE, Dayvyd et al. Adesão ao tratamento do diabetes mellitus e hipertensão arterial sistêmica: um enfoque nas relações de gênero. Revista de Enfermagem UFPE Online, Recife, v. 5, n. 10, p. 2359-2367, dez. 2011. Disponível em: https://periodicos.ufpe.br/ revistas/revistaenfermagem/article/download/6952/6201. Acesso em: 11 nov. 2016.

ANDRADE, Luciara et al. Relação da autopercepção de saúde, capacidade funcional e condições de saúde de idosos longevos residentes em domicílio em Jequié-BA. Estudos Interdisciplinares sobre o Envelhecimento, Porto Alegre, v. 23, n. 1, p. 75-86, 2018.

ASSOCIAÇÃO BRASILEIRA DE EMPRESAS DE PESQUISA (ABEP). Critérios de Classificação Econômica Brasil. São Paulo: ABEP, 2014. Disponível em: http://www.abep. org/Servicos/Download.aspx?id=12. Acesso em: 14 nov. 2016.

BOAVENTURA, Lara et al. UMI: Universidade da Melhor Idade. In: SEMINÁRIO DE EXTENSÃO UNIVERSITÁRIA DA REGIÃO CENTRO-OESTE, 5., 2012, Goiânia. Anais [...]. Goiânia, UFG: 2012. p. 45-51.

BORIM, Flávia; BARROS, Marilisa; NERI, Anita. Autoavaliação da saúde em idosos: pesquisa de base populacional no Município de Campinas, São Paulo, Brasil. Cadernos de Saúde Pública, Rio de Janeiro, v. 28, n. 4, p. 769-780, abr. 2012. Disponível em: http://unicamp.sibi.usp.br/bitstream/handle/SBURI/36331/S0102-311X2012000400016. pdf?sequence=1. Acesso em: 12 nov. 2016

CACHIONI, Meire. Universidade da Terceira Idade. In: NERI, Anita Liberalesso (org.). Palavras-chave em Gerontologia. Campinas: Alínea, 2005. p. 207-210.

CONFORTIN, Susana et al. Autopercepção positiva de saúde em idosos: estudo populacional no Sul do Brasil. Cadernos de Saúde Pública, Rio de Janeiro, v. 31, n. 5, p. 1049-1060, maio 2015.

COSTA, Clarisse et al. Utilização de medicamento pelos usuários da atenção primária do Sistema Único de Saúde. Revista de Saúde Pública, São Paulo, v. 51, n. 2, p. 11s, 2017.

COSTA, Francine; NAKATA, Priscila; MORAIS, Eliane. Estratégias desenvolvidas pelos idosos residentes na comunidade para morarem sozinhos. Texto Contexto Enfermagem, Florianópolis, v. 24, n. 3, p. 818-825, jul./set. 2015.

DÁTILO, Gilsenir; BRAGA, Mariana. Inclusão social da pessoa idosa no contexto acadêmico: a importância da Universidade Aberta da Terceira Idade como possibilidade de Educação Permanente ao idoso. In: JORNADA DO NúCLEO DE ENSINO DE MARÍLIA, 14., 2015, Marília. 14ª Jornada Pedagógica: anais. Marília: Editora da Unesp de Marília, 2015. p. 9.

DÁTILO, Gilsenir; MARIN, Maria. O envelhecimento na percepção de idosos que frequentam uma universidade aberta da terceira idade. Revista Estudos Interdisciplinares sobre o Envelhecimento, Porto Alegre, v. 20, n. 2, p. 597-609, 2015. 
FARIA, Magda; DAVID, Helena; ROCHA, Patrícia. Inserção e prática religiosa entre mulheres: aspectos protetores ao uso de álcool e violência. Revista Eletrônica de Saúde Mental Álcool e Drogas, São Paulo, v. 7, n. 1, p. 32-37, 2011. Disponível em: http://pepsic. bvsalud.org/scielo.php?script=sci_arttext\&pid=\$1806-69762011000100006\&lng=pt\& tlng=pt. Acesso em: 17 nov. 2016.

GIROTTO, Edmarlon et al. Adesão ao tratamento farmacológico e não farmacológico e fatores associados na atenção primária da hipertensão arterial. Ciência e Saúde Coletiva, Rio de Janeiro, v. 18, n. 6, p. 1763-1772, jun. 2013.

HAYMUSSI, Hilevi. Políticas públicas voltadas ao idoso, com recorte na educação: o programa Universidade Aberta da Maior Idade (UAMI)/UNIARP/Caçador. Revista Professare, Caçador, v. 4, n. 2, p. 51-76, 2015.

INOUYE, Keika et al. Efeito da Universidade Aberta à Terceira Idade sobre a qualidade de vida do idoso. Educação e Pesquisa, São Paulo, v. 44, p. e142931, 2018.

KINOSHITA, Larissa et al. Doenças autorrelatadas e satisfação com a vida de participantes da Universidade Aberta à Terceira Idade da Escola de Artes Ciências e Humanidades da Universidade de São Paulo. Revista Temática Kairós Gerontologia, São Paulo, v. 15, n. 7, p. 107-128, dez. 2012.

LAURIA, Bianca; MALTA, Paula; DÁTILO, Gilsenir. O papel da Universidade Aberta da Terceira Idade - UNATI na inclusão educacional do idoso, possibilitando sua melhor qualidade de vida. In: JORNADA DO NÚCLEO DE ENSINO DE MARÍLIA, 15., 2015, Marília. Anais [...]. Marília: Editora da Unesp de Marília, 2015. p. 1-10. Disponível em: https://www.marilia.unesp.br/Home/Eventos/2015/jornadadonucleo/o-papel-dauniversidade-aberta.pdf. Acesso em: 17 nov. 2016.

LIMA-SILVA, Thaís et al. Universidade Aberta à Terceira Idade: como atrair novos Estudantes? Revista Temática Kairós Gerontologia, São Paulo, v. 15, n. 7, p. 259-276, dez. 2012

LOBO, Lígia. Polifarmácia entre os idosos de Dourados, Mato Grosso do Sul: um estudo de base populacional. 2015. Dissertação (Mestrado em Ciências da Saúde) - Universidade Federal da Grande Dourados, Dourados, 2015.

LOLLI, Maria; MAIO, Eliane. Uso da tecnologia por idosos: perfil, motivações, interesses e dificuldades. ECS, Sinop, v. 5, n. 2, p. 211-223, jul./dez. 2015.

MATTA, Samara et al. Fontes de obtenção de medicamentos por pacientes diagnosticados com doenças crônicas, usuários do Sistema Único de Saúde. Cadernos de Saúde Pública, Rio de Janeiro, v. 34, n. 3, p. e00073817, 2018.

MONTEIRO, Betânia; MONTEIRO, Magali. Estilo de vida e saúde de um grupo de idosos de Franca, SP. Revista Geriatria e Gerontologia, Rio de Janeiro, v. 7, n. 2, p. 113-119, 2013.

MONTEIRO, Sally; AZEVEDO, Luzimeire; BELFORT, Illka. Automedicação em idosos em um Programa Saúde da Família, Brasil. Revista Infarma: Ciências Farmacêuticas, Brasília, v. 26, n. 2, p. 90-95, 2014.

MORISKY, Donald; GREEN, Lawrence; LEVINE, David. Concurrent and predictive validity of a self-reported measure of medication adherence. Medical Care, New York, v. 24, n. 1, p. 67-74, 1986.

MUNIZ, Elaine et al. Análise do uso de medicamentos por idosos usuários de plano de saúde suplementar. Revista Brasileira de Geriatria e Gerontologia, Rio de Janeiro, v. 20, n. 3, p. 375-387, 2017.

NASCIMENTO, Jaqueline; VALDÃO, Giselle. Automedicação: educação para prevenção. Anais da Conferência Internacional de Estratégia em Gestão, Educação e Sistemas de Informação (CIEGESI): Gestão para o Mundo da Informação, Anápolis, v. 1, n. 1, p. 817, jun. 2012. Disponível em: http://www.anais.ueg.br/index.php/ciegesi/article/ view/1173. Acesso em: 11 nov. 2016. 
OLIVEIRA, Marcelo et al. Automedicação em idosos residentes em Campinas, São Paulo, Brasil: prevalência e fatores associados. Cadernos de Saúde Pública, Rio de Janeiro, v. 28, n. 2, p. 335-345, fev. 2012.

OLIVEIRA, Samanta et al. Perfil de medicamentos utilizados por automedicação por idosos atendidos em centro de referência. Einstein, São Paulo, v. 16, n. 4, p. eAO4372 nov. 2018. Disponível em: https://doi.org/10.31744/einstein_journal/2018AO4372. Acesso em: 17 nov. 2016

ORDONEZ, Tiago; CACHIONI, Meire. Motivos para frequentar um programa de educação permanente: relato dos alunos da universidade aberta à terceira idade da Escola de Artes, Ciências e Humanidades da Universidade de São Paulo. Revista Brasileira de Geriatria e Gerontologia, Rio de Janeiro, v. 14, n. 3, p. 461-474, 2011. ISSN 18099823. Disponível em: http://dx.doi.org/10.1590/S1809-98232011000300007. Acesso em: 17 nov. 2016.

PAULA JÚNIOR, José et al. Prática de polifarmácia por idosos cadastrados em unidade de atenção primária. Investigação, França, v. 13, n. 2, p. 15-18, 2013.

RIGHES, Cristina. Utilização de medicamentos por idosos, em um centro de convivência, no município de Campo Grande, MS. 2015. Dissertação (Mestrado em Saúde e Desenvolvimento da Região Centro Oeste) - Universidade Federal do Mato Grosso do Sul, Campo Grande, 2015.

RODRIGUES, Bruna. Avaliação do uso de medicamentos entre idosos atendidos em centros de referência em Manaus - AM. 2013. Dissertação (Mestrado em Saúde, Sociedade e Endemias na Amazônia) - Universidade Federal do Amazonas, Manaus, 2013.

RODRIGUES, Mineia. A configuração do lazer no espaço das universidades da terceira idade. Estudos Interdisciplinares sobre o Envelhecimento, Porto Alegre, v. 20, n. 3, p. $771-787,2015$.

ROQUE, Francelise et al. Perfil socioeconômico-cultural de uma universidade aberta à terceira idade: reflexo da realidade brasileira? Revista Brasileira de Geriatria e Gerontologia, Rio de Janeiro, v. 14, n. 1, p. 97-108, 2011.

ROZENDO, Adriano; BORTOLI, Suzana; DONADONE, Juliana. Reformulando práticas e conceitos na Terceira Idade: uma experiência da U3I/UFMT. Revista Temática Kairós Gerontologia, São Paulo, v. 17, n. 4, p. 175-187, dez. 2015

SANTOS, Adriana; NOGUEIRA, Dulcinéia; BORJA-OLIVEIRA, Caroline. Automedicação entre participantes de uma Universidade Aberta à Terceira Idade e fatores associados. Revista Brasileira de Geriatria e Gerontologia, Rio de Janeiro, v. 21, n. 4, p. 431-439, 2018.

SANTOS, Thalyta et al. Consumo de medicamentos por idosos, Goiânia, Brasil. Revista de Saúde Pública, São Paulo, v. 47, n. 1, p. 94-103, 2013.

SARDINHA, Ana et al. Adesão dos idosos com doenças crônicas ao tratamento medicamentoso. Revista de Pesquisa em Saúde, São Luís, v. 16, n. 3, p. 154-158, set./dez. 2015.

SMANIOTO, Francieli; HADDAD, Maria. Avaliação da farmacoterapia prescrita a idosos institucionalizados. Revista Brasileira de Enfermagem, Brasília, v. 66, n. 4, p. 523-527, 2013.

SOUZA, Marina et al. A vivência da sexualidade por idosas viúvas e suas percepções quanto à opinião dos familiares a respeito. Saúde e Sociedade, São Paulo, v. 24, n. 3, p. 936-944, 2015

STEFANO, Isabel et al. Uso de medicamentos por idosos: análise da prescrição, dispensação e utilização num município de porte médio do estado de São Paulo. Revista Brasileira de Geriatria e Gerontologia, Rio de Janeiro, v. 20, n. 5, p. 681-692, 2017.

TAVARES, Noemia et al. Fatores associados à baixa adesão ao tratamento medicamentoso em idosos. Revista de Saúde Pública, São Paulo, v. 47, n. 6, p. 1092-1101, 2013. 
VIANA, Karynna et al. Acesso a medicamentos de uso contínuo entre idosos, Brasil. Revista de Saúde Pública, São Paulo, v. 49, n. 14, p. 1-10, 2015.

WORLD HEALTH ORGANIZATION (WHO). Anatomical Therapeutic Chemical - ATC classification index with defined daily doses - DDD's. Olso: WHO, 2013.

Data de Submissão: 17/12/2016

Data de Aprovação: 12/05/2020 
